\title{
Acute Cardiac Injury in COVID-19: A Systematic Review and Meta-analysis
}

\author{
Mehrbod Vakhshoori, MD"*; Maryam Heidarpour, MD²"; Davood Shafie, MD ${ }^{1 *}$; Marzieh Taheri, PhD ${ }^{3}$; Nima Rezaei, MD ${ }^{4,5,6}$; \\ Nizal Sarrafzadegan, $\mathrm{MD}^{7}$

\begin{abstract}
${ }^{1}$ Heart Failure Research Center, Cardiovascular Research Institute, Isfahan University of Medical Sciences, Isfahan, Iran
${ }^{2}$ Isfahan Endocrine and Metabolism Research Center, Isfahan University of Medical Sciences, Isfahan, Iran

${ }^{3}$ Interventional Cardiology Research Center, Cardiovascular Research Institute, Isfahan University of Medical Sciences, Isfahan, Iran

${ }^{4}$ Research Center for Immunodeficiencies, Children's Medical Center, Tehran University of Medical Sciences, Tehran, Iran

${ }^{5}$ Department of Immunology, School of Medicine, Tehran University of Medical Sciences, Tehran, Iran

${ }^{6}$ Network of Immunity in Infection, Malignancy and Autoimmunity (NIIMA), Universal Scientific Education and Research Network (USERN), Tehran, Iran

${ }^{7}$ Isfahan Cardiovascular Research Center, Cardiovascular Research Institute, Isfahan University of Medical Sciences, Isfahan, Iran
\end{abstract}

\begin{abstract}
Background: Coronavirus disease 2019 (COVID-19) has been widespread since late December 2019, with several symptoms related to the upper and lower respiratory system. However, its cardiac manifestations are less frequently studied. We aimed to analyze the available COVID-19 data on acute cardiac injury, using troponin and brain natriuretic peptide (BNP) levels.

Methods: We performed a systematic review on Medline/PubMed, Scopus, and Google Scholar databases until March 25, 2020. Relevant records reporting the incidence of acute cardiac injury as well as troponin and BNP levels were collected from published peer-reviewed articles with further analysis according to the clinical status of the patients (severe, non-severe, and death).

Results: Eleven records of 1394 individuals were included. The mean age of patients with acute cardiac injury was $56.6 \pm 33.4$ years (males: $54.3 \%)$. The incidence of acute cardiac injury was 15\% (95\% Cl: 11, 20\%). Further analysis revealed that dead or severe patients had significantly higher percentages of myocardial injury, compared to non-severe ones (peer-reviewed: 44\%, 95\% Cl: $16,74 \%$ vs. $24 \%, 95 \%$ Cl: $15,34 \%$ vs. $5 \%, 95 \%$ Cl: $1,12 \%$, respectively). Mean total troponin was $10.23 \mathrm{pg} / \mathrm{mL}(95 \% \mathrm{Cl}$ : 5.98, 14.47), while 13\% (95\% Cl: 8\%, 18\%) of patients had elevated levels. Mean BNP was 216.74 pg/mL (95\% Cl: 3.27, 430.20). Conclusion: Acute cardiac injury in COVID-19 patients is more frequent than what was expected at the beginning of the outbreak. Meanwhile, further studies are needed to investigate the utility of cardiac biomarkers as diagnostic and prognostic tools for longterm cardiac complications of this infection.

Keywords: Brain, Heart injuries, COVID-19, Coronavirus, Natriuretic peptide, Troponin

Cite this article as: Vakhshoori M, Heidarpour M, Shafie D, Taheri M, Rezaei N, Sarrafzadegan N. Acute cardiac injury in COVID-19: a systematic review and meta-analysis. Arch Iran Med. 2020;23(11):801-812. doi: 10.34172/aim.2020.107.
\end{abstract}

Received: May 12, 2020, Accepted: September 6, 2020, ePublished: November 1, 2020

\section{Introduction}

Infections due to Coronaviridiae have led to some of the most widespread disasters affecting the global population in the $21^{\text {st }}$ century. ${ }^{1}$ Severe acute respiratory syndrome (SARS) and the Middle East respiratory syndrome (MERS) are two members of this group, leading to hazardous epidemics in 2002 and 2012 with 10\% and 37\% mortality rates, respectively. ${ }^{2-5}$ In December 2019, several cases of pneumonia of unknown etiology were reported in Wuhan, Hubei, China. ${ }^{6}$ Prompt analysis of respiratory tract samples identified a virus with similar characteristics to the Coronaviridiae family with $79.5 \%$ identical genome to SARS; a new virulent virus which was initially named the 2019 novel coronavirus (2019-nCoV), results in SARS coronavirus 2 (SARS-CoV-2), also named the coronavirus disease 2019 (COVID-19)..$^{7-10}$ Since that time, more than 3 million cases have been reported all over the world at an incredibly rapid rate in a way that the term "pandemic" was used by the World Health Organization (WHO) to describe it in March 2020. Clinical manifestations of this disease among diagnosed patients range from mild to severe. This disease manifests primarily with nonspecific symptoms, including fatigue, myalgia, and fever. However, this infection can further involve pulmonary tracts, causing its specific respiratory symptoms, including dry cough, dyspnea, or even acute respiratory distress. ${ }^{5,10-12}$

In addition to its distinctive site of infection, this highly contagious disease can also affect other parts of the body, including cardiac involvement which has been reported as one of its complications with different reported prevalence 
rates. 5,10,12,13 Therefore, implementing a meta-analysis with the integration of previously published studies seems necessary.

We aimed to analyze the studies reporting acute cardiac injury as well as the alteration in cardiac biomarkers related to this injury, including troponin and brain natriuretic peptide (BNP) in patients infected with the COVID-19 infection.

\section{Materials and Methods}

\section{Protocol and Registration}

This study has been designed in the context of Preferred Reporting Items for Systematic Reviews and MetaAnalyses (PRISMA) statement. ${ }^{14}$ Furthermore, the current manuscript has been registered in the International Prospective Register of Systematic Reviews (PROSPERO) database registry (CRD42020176685).

\section{Eligibility Criteria}

In order to investigate acute myocardial injury and cardiac laboratory abnormalities [including troponin/ hypersensitive troponin and proBNP or N-terminal proBNP (NT-proBNP)] of patients confirmed with COVID-19 infection, we included all published peerreviewed case series, cross-sectional, review, cohort and case-control studies without any language limitations. We further excluded non-peer reviewed articles, animal studies, case reports, letters without reporting patients' characteristics, or any kind of studies with incomplete information.

\section{Information Source and Search Strategies}

We performed a systematic review using Medline/PubMed, Scopus, and google scholar databases without any time limitations for the first two. However, articles from Jan 1, 2020 were included in our search in the google scholar database. For assessment of cardiac injury, we searched the following terms, "acute cardiac injury”, "acute myocardial injury”, "acute heart injury”, "cardiac injury”, "myocardial injury", "heart injury", "SARS-CoV-2", "coronavirus", "covid-19", "2019-nCoV" and "coronavirus covid-19". The following terms in the above-mentioned databases were used in combination for investigating myocardial laboratory enzymes abnormalities, "cardiac troponin", "troponin”, “c-TnI”, "troponin I", "cardiac biomarkers", "cardiac markers", "cardiac laboratory", "brain natriuretic peptide”, "BNP”, "proBNP”, "NT-proBNP”, “SARSCoV-2", "coronavirus", "covid-19", "2019-nCoV” and "coronavirus covid-19".

\section{Data Management and Selection Process}

Figure 1 shows the flow diagram of the current study according to our pre-defined variables, including acute cardiac injury, troponin, and BNP (Figure $1 \mathrm{~A}, \mathrm{~B}$, and C, respectively). Two independent reviewers first screened the titles and abstracts of articles. If they were relevant, full texts would be obtained and assessed further based on inclusion and exclusion criteria. In terms of duplicate publications, only one was considered. In the presence of any terms indicating cardiac injury or myocardial enzymes, including troponin and BNP, the articles were selected and classified in their specific groups.

\section{Data Collection Process and Data Items}

Data including authors' names, date of study, sample size, age (median and interquartile range (IQR) or mean \pm standard deviation, ${ }^{15}$ as reported), sex (male/female), myocardial complication (acute cardiac injury), laboratory information (including troponin/hypersensitive troponin and proBNP or NT-proBNP) as well as proportions of elevated troponin and BNP were extracted by two independent investigators, as appropriate. The third researcher assessed the probable discrepancies between data extraction files, and any disagreements were resolved by consensus.

\section{Risk of Bias Assessment, Data Synthesis, and Statistical Approach}

To assess the quality of cross-sectional studies, a critical appraisal tool (AXIS tool) was utilized. ${ }^{16}$ In terms of other observational studies or systematic reviews, we used "strengthening the reporting of observational studies in epidemiology" (STROBE) or "assessment of multiple systematic reviews" (AMSTAR) tools, as appropriate. ${ }^{17,18}$ Both pooled results of proportions and means (MRAW: mean raw) with their respective 95\% CIs were calculated using inverse-variance with the random-effects model, for which DerSimonian-Laird estimator was used for tausquared $\left(\tau^{2}\right)$. The statistical method suggested by Wan and colleagues was used for estimation of means, and SDs in studies reported medians and IQRs for continuous variables. ${ }^{19}$ Forest plots were utilized to show the impact of COVID-19 on acute cardiac injury and specific myocardial biomarkers, including troponin and BNP. All unit variables were converted to standard units for better analysis. Cochran's $\mathrm{Q}$ statistic, $\mathrm{I}^{2}$ and the $\tau^{2}$ were used to measure heterogeneity. Fixed and random-effect models were used according to the heterogeneity of outcomes, as appropriate. We further divided the study population based on the severity of the disease reported by each study. We analyzed subgroups of each pre-defined variables as severe group (including intensive care unit (ICU), critically ill, dangerous, intubated, and severe), non-severe group (including non-ICU, non-critical, ordinary, non-severe and not-intubated) and death group (including nonsurvivor and expired) accordingly. All analyses were done using $\mathrm{R}$ software (version 3.6.3) meta-package (version 4.11-0) as well as STATA (version 14), and P-values less than 0.05 were considered statistically significant. 
Figure 1. Flow diagram of studies (A: acute cardiac injury, B: troponin, C: BNP).

See Figure $1 \mathrm{C}$ in the next page.
(A)

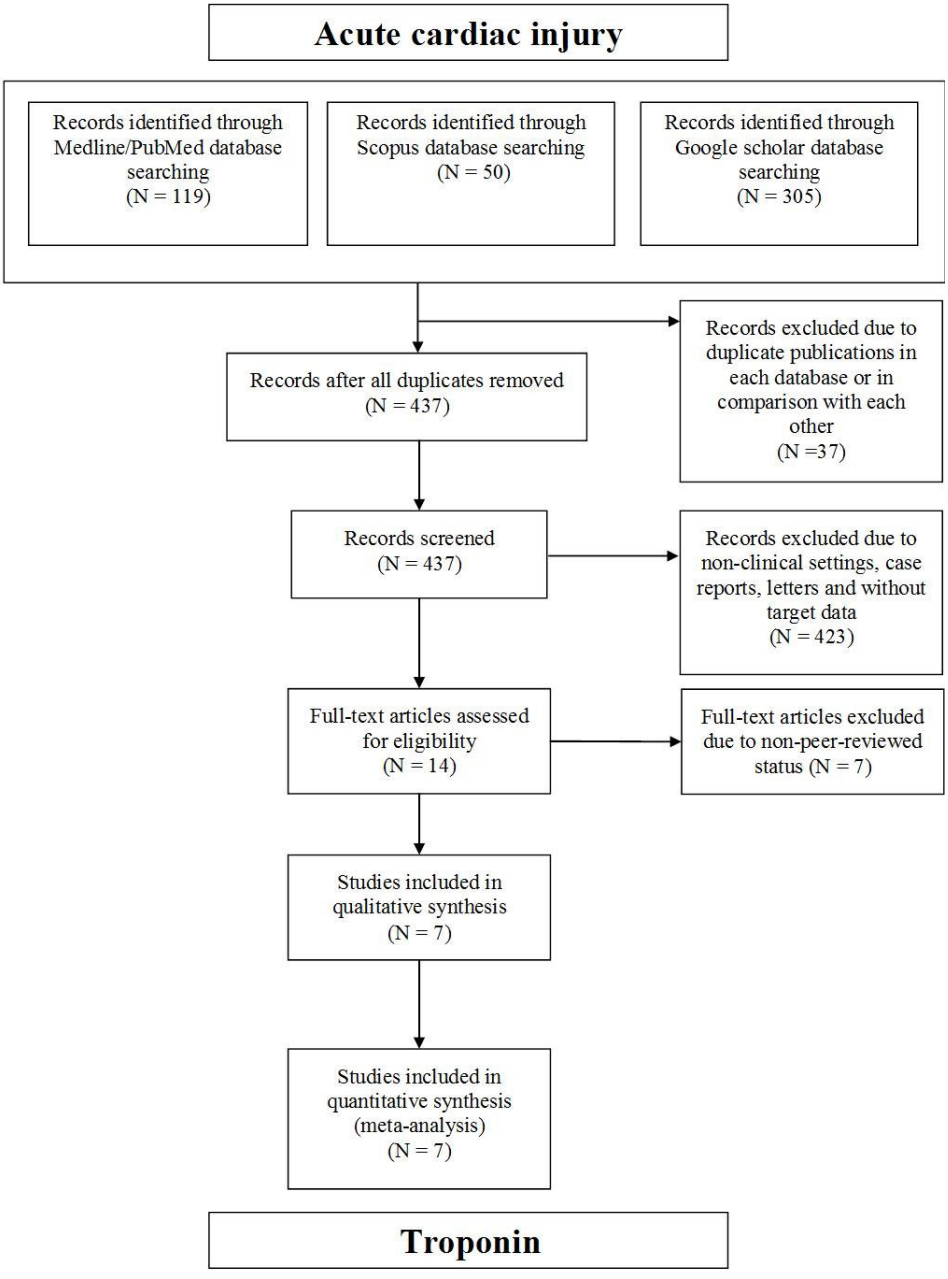

(B)

\begin{tabular}{|c|c|c|}
\hline $\begin{array}{c}\text { Records identified through } \\
\text { Medline/Pubmed database } \\
\text { searching } \\
(\mathrm{N}=118)\end{array}$ & $\begin{array}{c}\text { Records identified through } \\
\text { Scopus database searching } \\
(\mathrm{N}=6)\end{array}$ & $\begin{array}{c}\text { Records identified through } \\
\text { Google scholar database } \\
\text { searching } \\
(\mathrm{N}=126)\end{array}$ \\
& \\
\end{tabular}

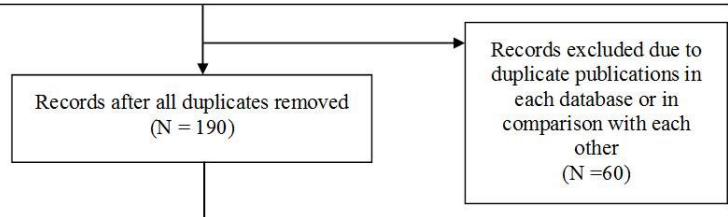

Records excluded due to non-clinical settings, case reports, letters and without target data

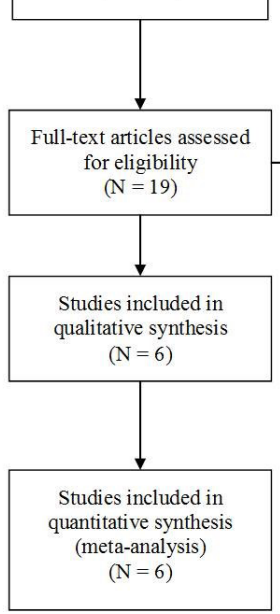




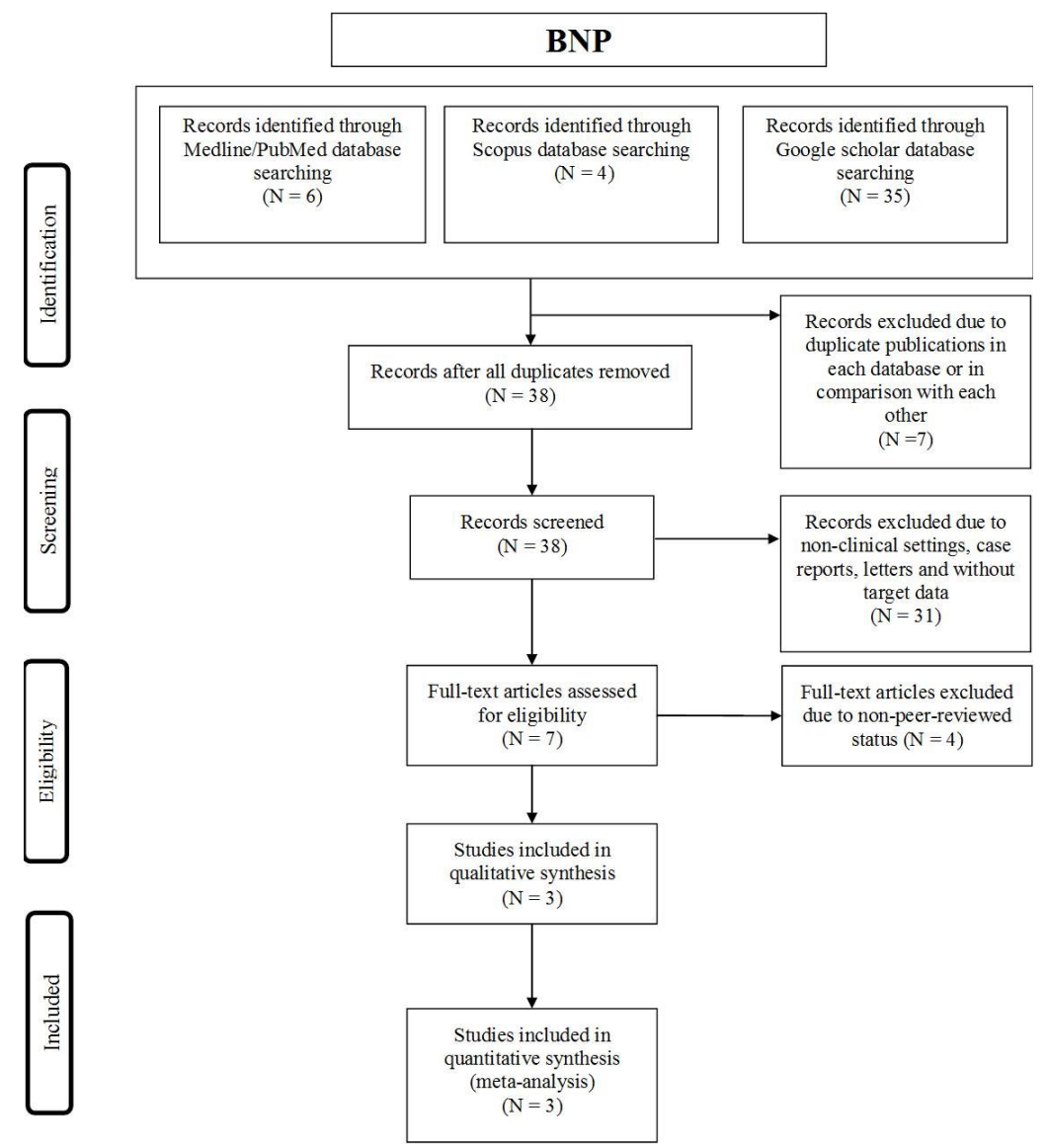

Figure 1. Flow diagram of studies (A: acute cardiac injury, B: troponin, C: BNP).

\section{Results}

Study Selection and Characteristics

A total of 474 records were found in databases; among them 37 were duplicates either in one database or several. We further excluded 423 articles due to various reasons, including animal studies, non-clinical settings, or other study types, which we had pre-defined as exclusion criteria. As a result, the full-texts of 14 articles were retrieved with the further exclusion of seven due to the non-peer-reviewed status of manuscripts (Figure 1A). Acute cardiac injury was defined as either elevation of cardiac troponin above the $99^{\text {th }}$ percentile of upper normal limit or recent electrocardiographic or echocardiographic abnormalities. 5,12,20,21 Regarding troponin, our primary search revealed 250 records; 190 of them were selected after removal of duplicates. After exclusion of 171 more manuscripts, the full-texts of 19 , including six peerreviewed articles were obtained for our further analysis (Figure 1B). Forty-five articles were found during the search on BNP levels among patients infected with COVID-19. After the removal of 7 duplicates and assessment of the other 38 records, three peer-reviewed studies were eligible for data extraction and analysis (Figure 1C).

The summary characteristics and analysis of all included studies are shown in Tables 1 and 2. All studies were performed in China and had cross-sectional designs. The total number of patients, according to each category, was as follows: 970 for acute cardiac injury, 682 for troponin, and 678 for BNP. Risk of bias assessment of all included studies is shown in Table S1.

Acute Cardiac Injury

The mean age of participants was $56.6 \pm 33.4$ years (males: $54.3 \%)$. Forest plots for proportions of acute cardiac injury are depicted in Figure 2. Our analysis revealed that 15\% (95\% CI: 11\%, 20\%) of individuals infected with COVID-19 suffered from acute cardiac injury (Figure 2A). Further subgroup analysis showed that myocardial injury was less prevalent in non-severe patients than either the severe or the death group $(5 \%, 95 \% \mathrm{CI}: 1,12 \%$, vs. $24 \%$, $95 \%$ CI: $15 \%$, 34\%, vs. $44 \%$, $95 \%$ CI: $16 \%$, $74 \%$, respectively) (Figure $2 \mathrm{~B}$ ). Moreover, the analysis showed that severe patients were at 4.74 times (95\% CI: 2.30, 9.78) higher risk of having an acute cardiac injury in comparison to non-severe subjects (Figure 3).

\section{Troponin}

Patients had a mean age of $54.5 \pm 15.1$ years, and $56.5 \%$ of them were men. Forest plots, as shown in Figure 4, revealed that the mean high-sensitive troponin (hs-troponin) as well as troponin I/T among all study population was $6.81 \mathrm{pg} /$ $\mathrm{mL}(95 \%$ CI: 4.47, 9.16) and $30.49 \mathrm{pg} / \mathrm{mL}$ (95\% CI: 0.84, 


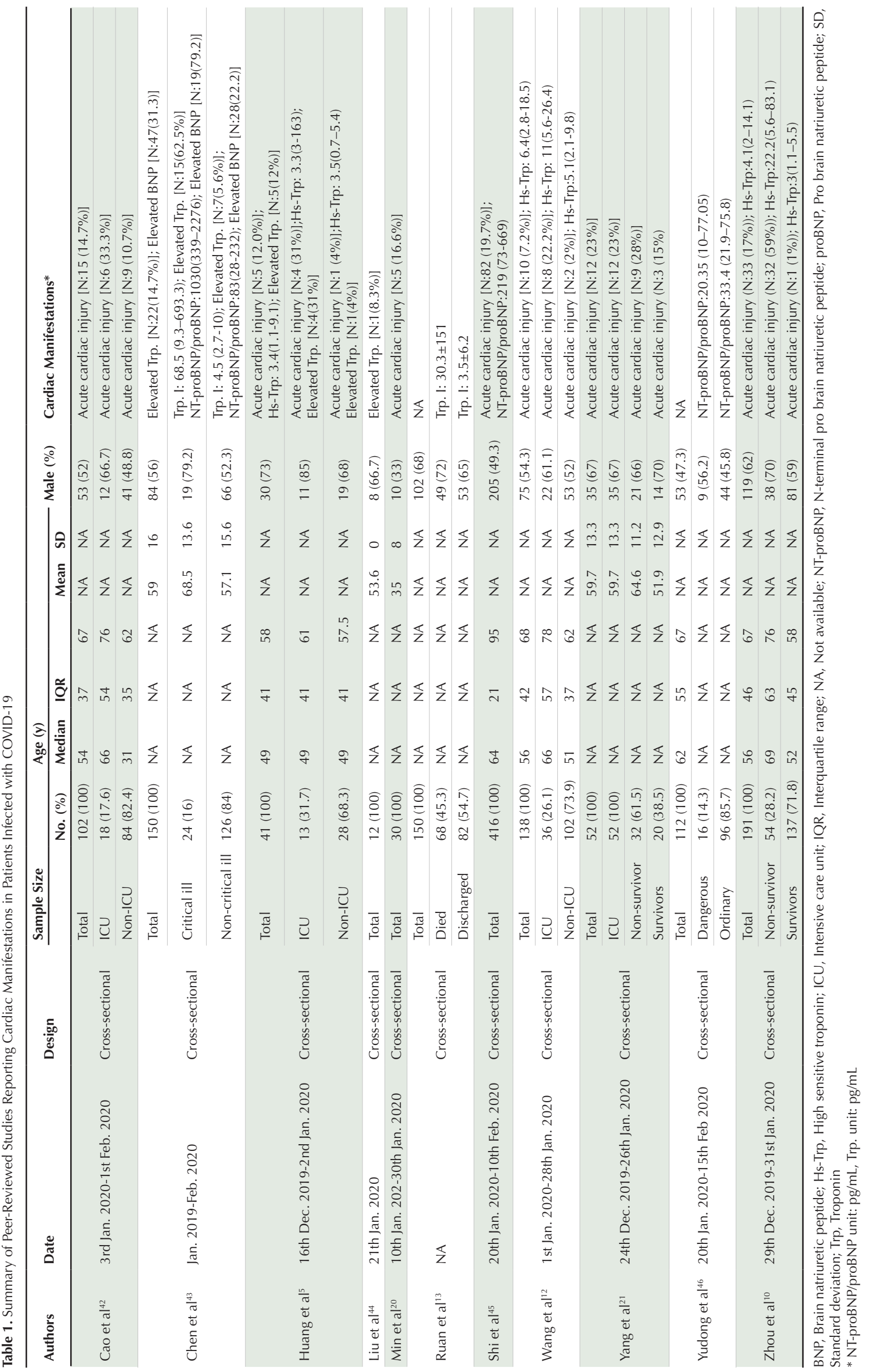


Table 2. Summary Analysis Result of all Peer- and Non-Peer-Reviewed Studies According to Acute Cardiac Injury, Troponin, and BNP

\begin{tabular}{|c|c|c|c|c|c|c|c|}
\hline Cardiac Manifestation & Population & $\mathbf{Q}^{*}$ & $I^{2 * *}$ & $\tau^{2^{* * *}}$ & $P$ & Proportions & $95 \%$ Confidence Interval \\
\hline \multirow{2}{*}{ Acute cardiac injury } & Total & 16.22 & $63 \%$ & 0.0035 & 0.01 & 0.15 & $0.11,0.20$ \\
\hline & Subgroups & 89.14 & $91 \%$ & 0.0618 & $<0.01$ & 0.20 & $0.07,0.36$ \\
\hline \multirow{2}{*}{ Elevated troponin } & Total & 0.2 & 0 & 0 & 0.90 & 0.13 & $0.08,0.18$ \\
\hline & Subgroups & 11.26 & $93 \%$ & 0.0935 & $<0.01$ & 0.21 & $0.01,0.52$ \\
\hline \multirow{2}{*}{ Elevated BNP } & Total & - & - & - & - & - & - \\
\hline & Subgroups & - & - & - & - & - & - \\
\hline Cardiac Manifestation & Population & Q & $I^{2}$ & $\mathrm{~T}^{2}$ & $P$ & MRAW & $\mathbf{9 5} \%$ Confidence Interval \\
\hline \multirow{2}{*}{ Hs-Troponin } & Total & 11.48 & $83 \%$ & 3.5221 & $<0.01$ & 6.81 & $4.47,9.16$ \\
\hline & Subgroups & 38.02 & $89 \%$ & 12.6034 & $<0.01$ & 8.84 & $4.65,13.04$ \\
\hline \multirow{2}{*}{ Troponin $\mathrm{I} / \mathrm{T}$} & Total & 10.6 & $91 \%$ & 414.6549 & $<0.01$ & 30.49 & $0.84,60.13$ \\
\hline & Subgroups & 7.01 & $71 \%$ & 818.6549 & 0.03 & 24.54 & $-17.58,66.67$ \\
\hline \multirow{2}{*}{ Total troponin } & Total & 44.42 & $91 \%$ & 14.8197 & $<0.01$ & 10.23 & $5.98,14.47$ \\
\hline & Subgroups & 46.29 & $85 \%$ & 6.3467 & $<0.01$ & 7.26 & $4.60,9.93$ \\
\hline \multirow{2}{*}{ BNP } & Total & 220.45 & $99 \%$ & 35127.3004 & $<0.01$ & 216.74 & $3.27,430.20$ \\
\hline & Subgroups & 0.74 & $92 \%$ & 1919.4907 & $<0.01$ & 71.89 & $20.89,122.90$ \\
\hline
\end{tabular}

* Cochran's Q statistic for heterogeneity; ** Index for the degree of heterogeneity; *** Tau-squared measure of heterogeneity.

BNP, Brain natriuretic peptide; Hs-Troponin, High sensitive troponin; MRAW, Mean raw.

(A)

Study

Wang et al.2020
Yang et al. 2020
Huang et al. 2020
Min et al.2020
Zhou et al.2020
Cao et al.2020
Shi et al.2020

Random effects model
Events Total

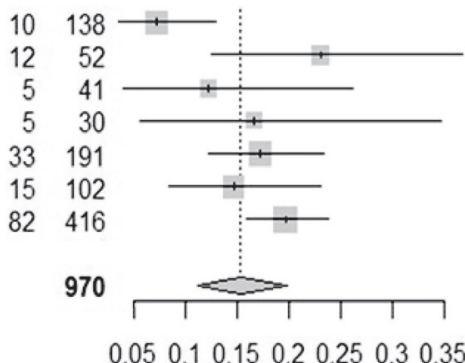

$\begin{array}{llllll}0.05 & 0.1 & 0.15 & 0.2 & 0.25 & 0.30 .35\end{array}$
Proportion $\quad 95 \%-\mathrm{Cl}$ Weight

$0.07[0.04 ; 0.13] \quad 16.8 \%$

$0.23[0.13 ; 0.37] \quad 10.8 \%$

$0.12[0.04 ; 0.26] \quad 9.4 \%$

$0.17[0.06 ; 0.35] \quad 7.7 \%$

$0.17[0.12 ; 0.23] \quad 18.6 \%$

$0.15[0.08 ; 0.23] \quad 15.0 \%$

$0.20[0.16 ; 0.24] \quad 21.7 \%$

$0.15[0.11 ; 0.20] 100.0 \%$

Heterogeneity: $\digamma^{2}=63 \%, \tau^{2}=0.0035, \rho=0.01$

(B)

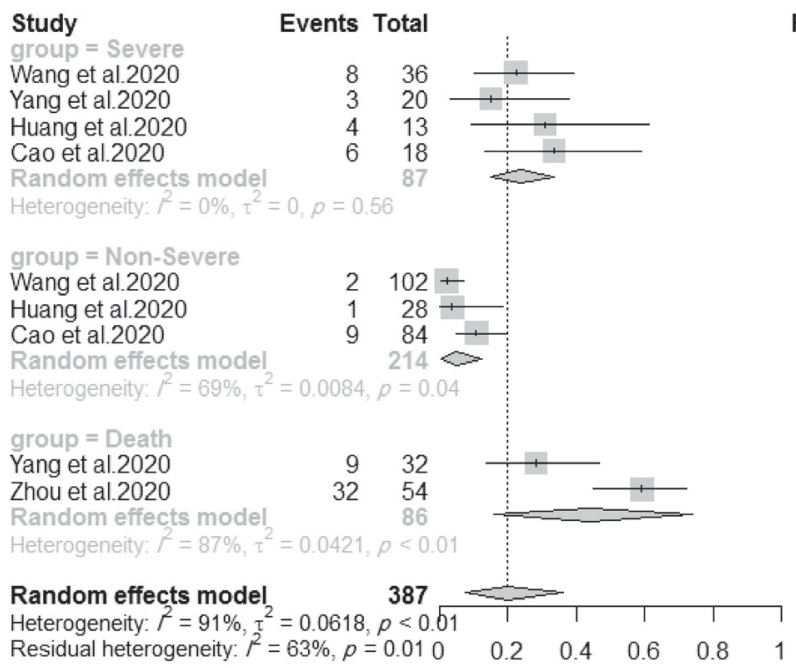

Proportion $\quad 95 \%-\mathrm{Cl}$ Weight

$0.22[0.10 ; 0.39] \quad 11.3 \%$

$0.15[0.03 ; 0.38] \quad 10.5 \%$

$0.31[0.09 ; 0.61] \quad 9.7 \%$

$0.33[0.13 ; 0.59] \quad 10.3 \%$

$0.24[0.15 ; 0.34] \quad 41.9 \%$

$0.02[0.00 ; 0.07] \quad 12.1 \%$

$0.04[0.00 ; 0.18] \quad 11.0 \%$

$0.11[0.05 ; 0.19] \quad 12.0 \%$

$0.05[0.01 ; 0.12] \quad 35.2 \%$

$0.28[0.14 ; 0.47] \quad 11.2 \%$

$0.59[0.45 ; 0.72] \quad 11.7 \%$

$0.44[0.16 ; 0.74] \quad 22.9 \%$

$0.20[0.07 ; 0.36] 100.0 \%$

Figure 2. Forest Plots for Proportions of Acute Cardiac Injury Based on Total (A) and Subgroups (B) of Studies. 


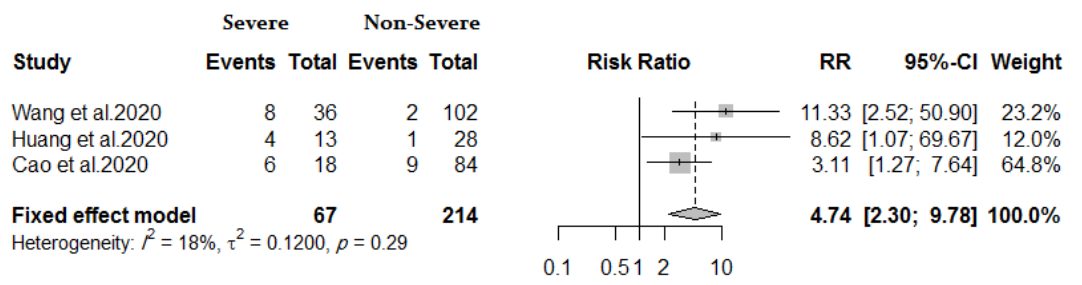

Figure 3. Forest Plot for the Risk Ratio of Acute Cardiac Injury According to Severe and Non-Severe Groups.

60.13), respectively (Figures $4 \mathrm{~A} \& 4 \mathrm{~B}$, respectively). Mean total troponin, including both hs-troponin and troponin I/T, was $10.23 \mathrm{pg} / \mathrm{mL}$ (95\% CI: 5.98, 14.47) (Figure 4C). Moreover, $13 \%$ (95\% CI: $8 \%, 18 \%)$ of patients had elevated troponin levels (Figure 4D). Further subgroup analysis of hs-troponin showed that the mean level in the severe group was $19.33 \mathrm{pg} / \mathrm{mL}$ (95\% CI: -7.37, 46.03). The mean values in non-severe and death groups were $4.47 \mathrm{pg} / \mathrm{mL}$ (95\% CI: 2.05, 6.89) and $36.97 \mathrm{pg} / \mathrm{mL}(95 \%$
CI: 21.23, 52.71), respectively (Figure 4E). In terms of troponin I/T, the results were the followings: severe group: $257.03 \mathrm{pg} / \mathrm{mL}$ (95\% CI: 41.38, 472.68), non-severe group: $5.73 \mathrm{pg} / \mathrm{mL}(95 \% \mathrm{CI}: 4.77,6.69)$ and death group: 30.3 pg/mL (95\% CI: -5.59, 66.19) (Figure 4F). Nonsevere individuals had lower mean values of total troponin (including hs-troponin and troponin I/T) compared to deceased subjects $(4.93 \mathrm{pg} / \mathrm{mL}, 95 \% \mathrm{CI}: 3.45,6.41$ vs. 35.89 pg/mL, 95\% CI: 21.48, 50.31, respectively) (Figure

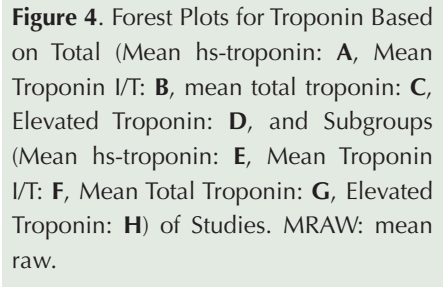

See Figure $4 \mathrm{E}-\mathrm{H}$ in the next page.
(A)

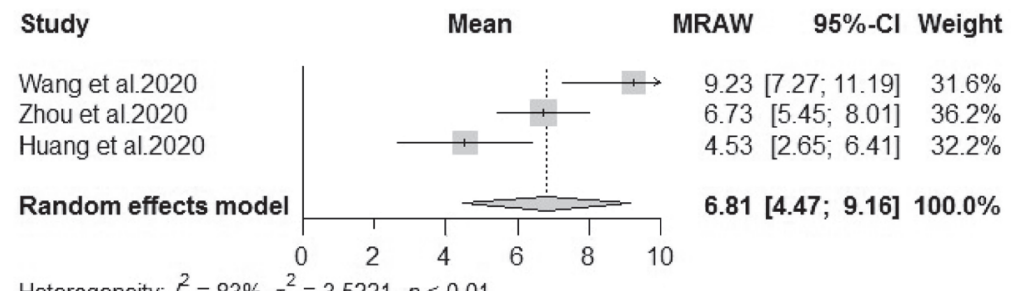

Heterogeneity: $\digamma^{2}=83 \%, \tau^{2}=3.5221, p<0.01$

(B)

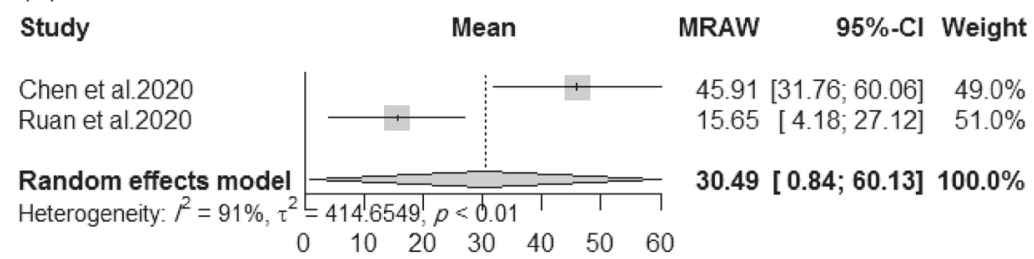

(C)

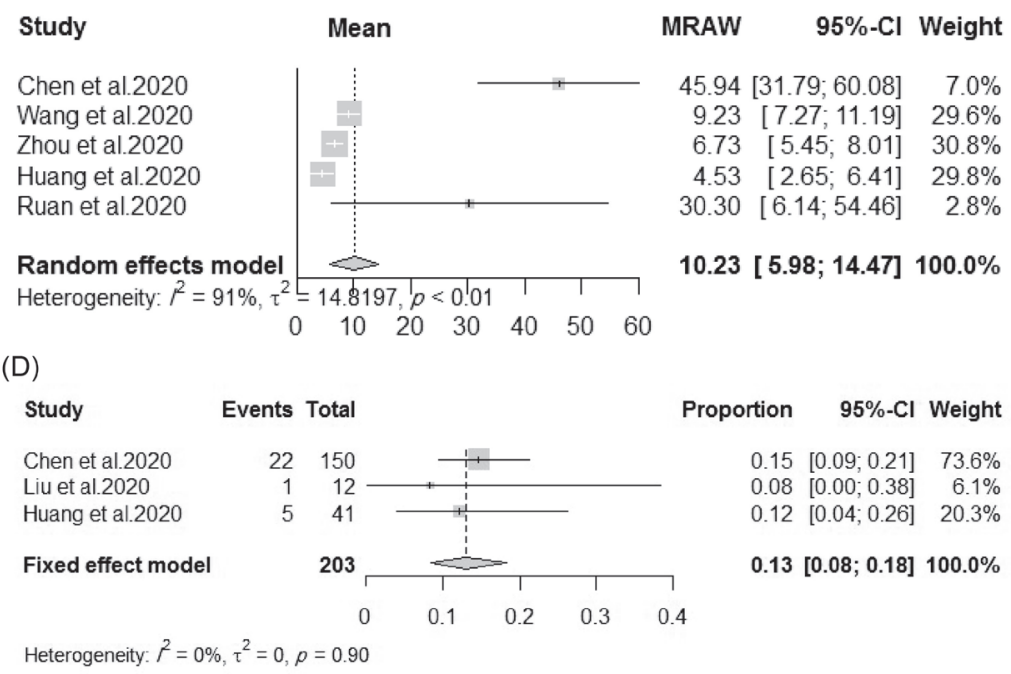




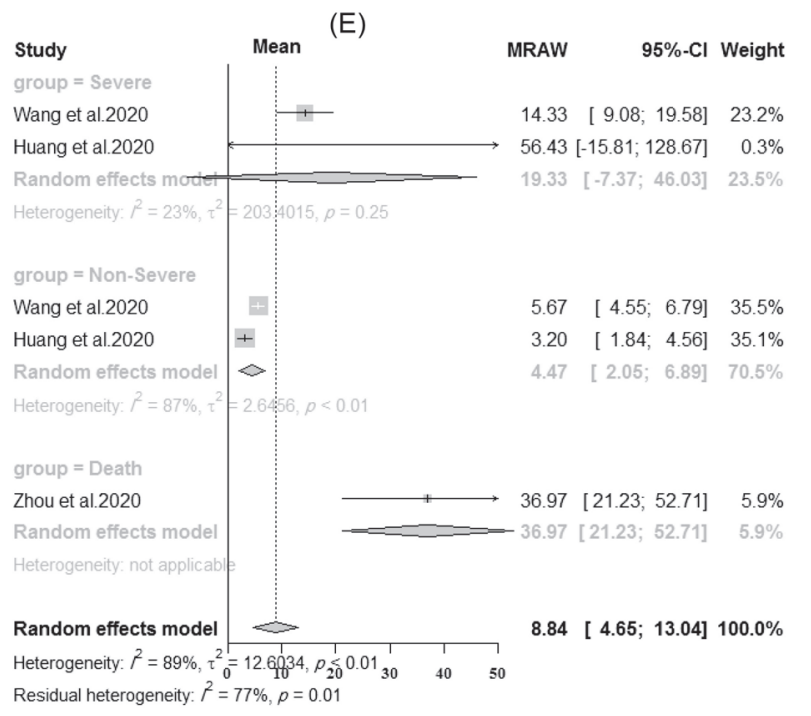

(G)
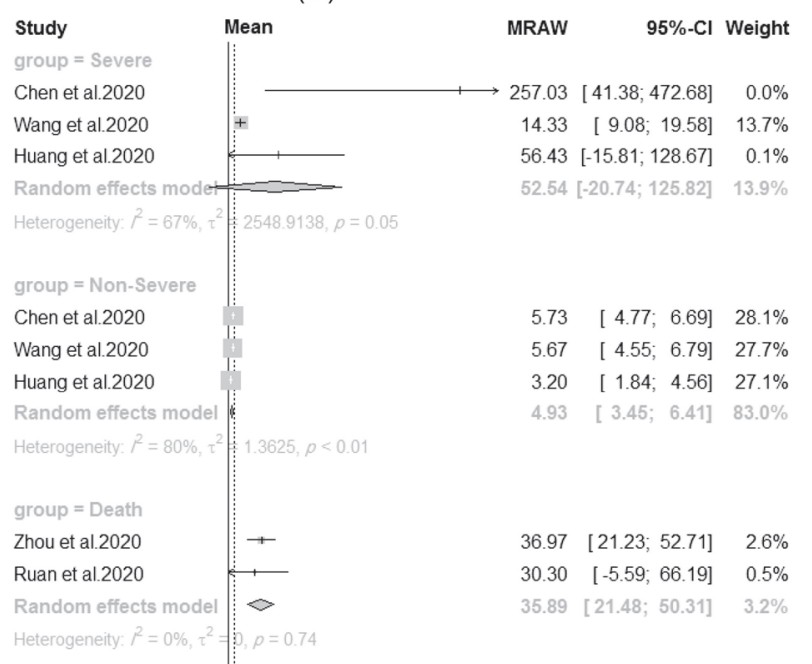

Random effects model

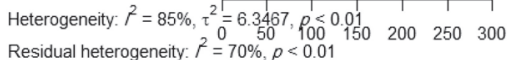

$7.26[4.60 ; 9.93] 100.0 \%$

Figure 4. Forest Plots for Troponin Based on Total (Mean hs-troponin: A, Mean Troponin I/T: B, mean total troponin: C, Elevated Troponin: D, and Subgroups (Mean hs-troponin: E, Mean Troponin I/T: F, Mean Total Troponin: G, Elevated Troponin: H) of Studies. MRAW: mean raw.

4G). Patients with severe manifestations had significantly higher percentages of elevated troponin levels compared to non-severe individuals ( $48 \%, 95 \%$ CI: $19 \%, 78 \%$ vs. $5 \%$ 95\% CI: 2\%, 9\%, respectively) (Figure 4H).

BNP

Participants had a mean age of $59.9 \pm 38.9$ years (males: $50.4 \%)$ with a mean level of $216.74 \mathrm{pg} / \mathrm{mL}$ (95\% CI: $3.27,430.20$ ) (Figure 5). Subgroup analysis showed that mean BNP values in the severe and non-severe groups were $584.31 \mathrm{pg} / \mathrm{mL}(95 \%$ CI: $-568.47,1737.10)$ and $77.82 \mathrm{pg} /$ $\mathrm{mL}$ (95\% CI: 8.64, 147), respectively (Figure 6).

\section{Discussion}

To the best of our knowledge, this study is the first in the literature to analyze all available data related to myocardial injury as well as cardiac biomarkers, including troponin
(F)

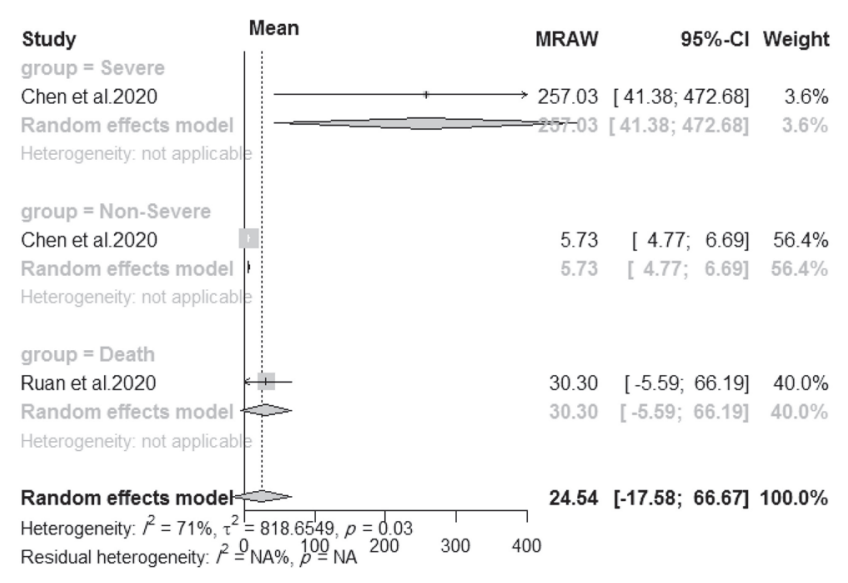

(H)

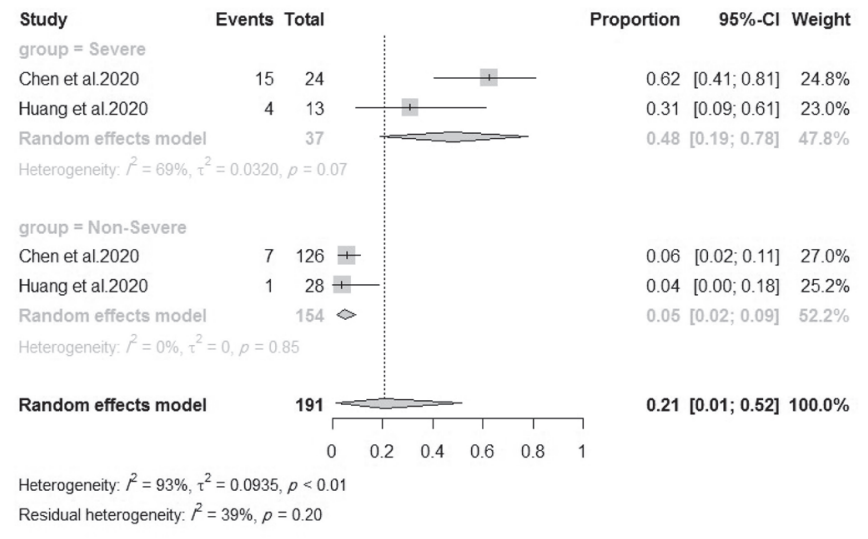

and BNP in patients infected with this highly contagious virus. We included data from a total of 1394 patients from all included records $(\mathrm{n}=11)$. We found out that cardiac injuries as well as myocardial biomarker abnormalities are quite prevalent among patients with the COVID-19 infection. Furthermore, patients experiencing the severe form of this infection are most likely at higher risk of cardiac tissue damage. Even though COVID-19 has some unspecific symptoms, including cough, fever, sore throat, and specific presentations related to the respiratory tract, cardiac manifestations are becoming a new considerable challenge, and development of new guidelines is required in this regard.

Our results showed that the frequency of acute cardiac injury was 15\% (95\% CI: 11, 20\%), with higher percentages among dead individuals (44\%, 95\% CI: 16 , 


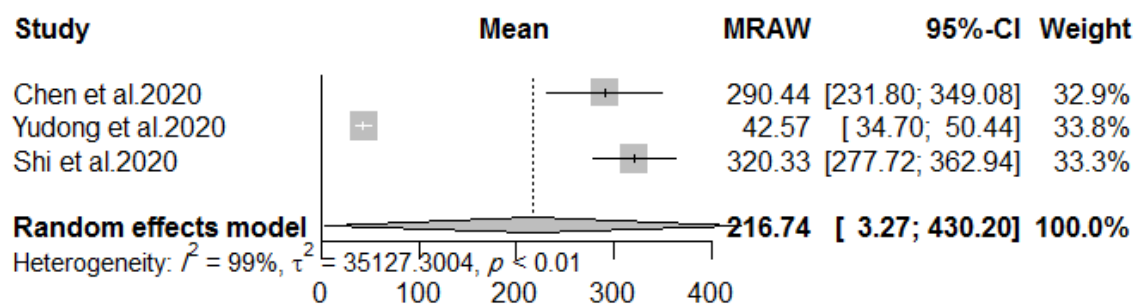

Figure 5. Forest Plots for NT-proBNP/proBNP Based on Total Studies. MRAW: mean raw.

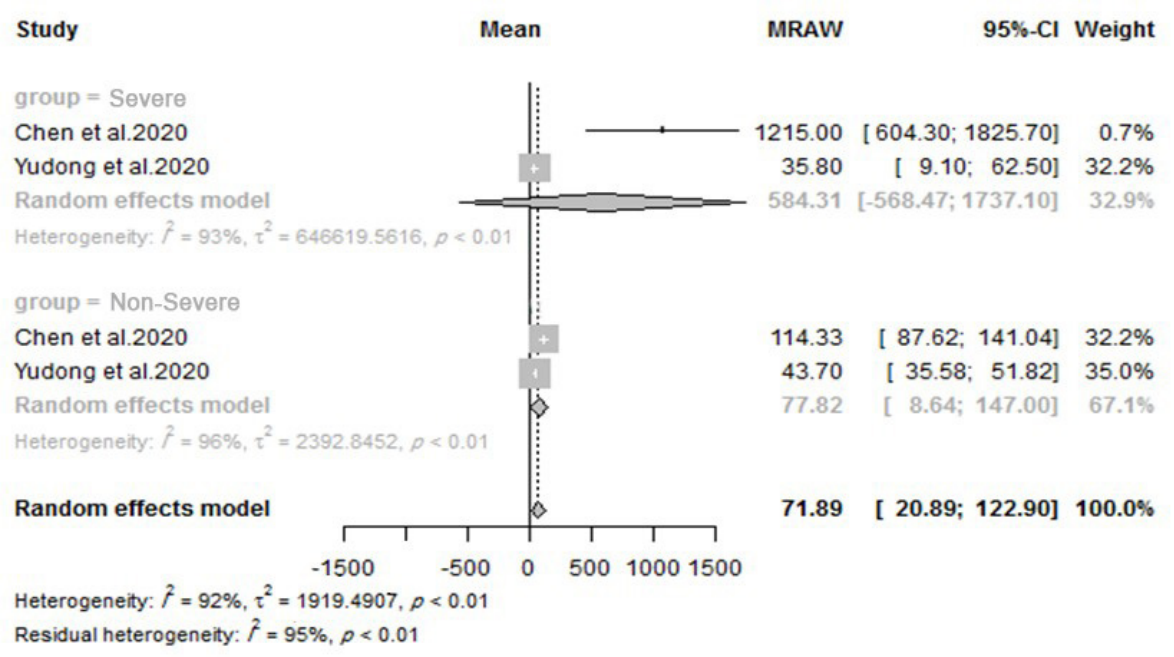

Figure 6. Forest Plots for NT-proBNP/proBNP Based on Subgroups of Studies. MRAW: mean raw.

$74 \%)$ or patients with the severe form of infection $(24 \%$, 95\% CI: 15, 34\%) who required intensive care. Although the exact mechanism of myocardial injury remains unknown, several mechanisms have been proposed. The first one is attributed to direct invasion of the virus to the myocardium through blood-borne access. A prior study on the SARS virus reported that $35 \%$ of cardiac tissues from deceased patients contained the RNA genome of this virus. ${ }^{22}$ Furthermore, angiotensin-converting enzyme 2 (ACE2) has been postulated as one of the primary receptors leading to cardiac damage. ${ }^{22,23}$ Oudit et al found that pulmonary infection with the human SARS virus in mice led to myocardial injury, which was related to decreased ACE2 levels. ${ }^{22}$ This target has been reported to be similar in the structure of binding domains with COVID-19. ${ }^{24}$ ACE2 is tissue-specific and expressed in the pulmonary, cardiovascular, gastrointestinal, and renal cells. ${ }^{23}$ Therefore, the high resemblance of SARS with the COVID-19 genome, as well as similarities in receptor binding domains, might lead to myocardial damage. Pneumonia induced by this virus causes hypoxemia and an imbalance between aerobic and anaerobic metabolism favoring the latter. This phenomenon would lead to intracellular acidosis and production of oxygen free radicals as well as the influx of calcium which ultimately results in myocyte injury and death. ${ }^{23}$ Another probable mechanism could be related to the cytokine storm. The imbalance between T helper 1 and 2 cells leads to enormous production of inflammatory cytokines which might play roles in the pathogenesis of cardiac injury. ${ }^{5}$ The possible side effects of antiviral agents, as well as development of the hypercoagulable state, might be other hypothetical mechanisms which need to be clarified. ${ }^{23,25}$

In our analysis, the mean level of total troponin was $10.23 \mathrm{pg} / \mathrm{mL}$ (95\% CI: 5.98, 14.47) and 13\% (95\% CI: $8,18 \%)$ of patients had elevated troponin levels. Besides, patients with severe disease had higher percentages of abnormal troponin ranges in comparison to individuals with a milder form of the disease. This biomarker has been suggested to be increased through several other mechanisms other than myocardial cell death, including increased cell membrane permeability or entry into blood of lower weight troponin fragments due to normal physiologic degradation. ${ }^{26,27}$ Although troponin has been previously suggested for the diagnosis of myocardial infarction due to coronary vessel obstruction, several other cardiac and non-cardiac disorders can also increase the plasma concentration of troponin. ${ }^{27}$ Thus, other possible secondary conditions other than injury of the cardiac tissue itself, must be taken into account. It has 
been reported that $12 \%-85 \%$ of patients admitted to ICU showed an elevation in troponin levels, which was associated with poor prognosis and increased mortality rates. ${ }^{15,27,28}$ Increased pulmonary vascular resistance (PVR) resulting from acute respiratory distress syndrome leading to extra strain on the right ventricle is proposed as another etiology of elevated troponin. ${ }^{27,29,30}$

Moreover, this biomarker could be raised by the administration of mechanical ventilation, which most patients require in ICU settings. ${ }^{29}$ Also, sepsis could be categorized as another possible etiology in this regard due to systemic inflammatory response syndrome and its specific manifestations due to oxygen supply-demand imbalance resulting from fever, increased body metabolism, and respiratory failure. ${ }^{27,31,32}$ Even tachycardia, either alone or in the context of sepsis, might be able to raise troponin levels..$^{27,33-35}$ It seems that the diagnosis of cardiac injury due to any increase in troponin must be interpreted with other clinical and para-clinical findings. Moreover, the cost-effectiveness of measurement of this marker as a prognostic factor must be assessed in future studies.

Our findings, in terms of BNP revealed that this biomarker had a mean level of $216.74 \mathrm{pg} / \mathrm{mL}(95 \%$ CI: 3.27, 430.20) and was more elevated among severe patients compared to individuals with the non-severe form of the disease. Nevertheless, due to quite small number of studies reporting this biomarker, interpretation of the results should be made with caution. Although increased BNP could aid in the diagnosis of heart failure, several other non-cardiac etiologies of this elevation should be considered. Advanced age, as well as anemia or renal failure, could elevate the level of this biomarker. ${ }^{36-38}$ Furthermore, right ventricle overload due to increased PVR in severe pulmonary diseases could partially play a role in the elevation of BNP. ${ }^{39}$ A diagnosis of myocardial damage solely based on this marker must be complemented with other relevant findings.

We tried our best to gather all relevant studies in this regard. However, several limitations may be attributed to this study. As COVID-19 data reporting is a daily process, we only analyzed appropriate studies by March 25, 2020. Lack of investigation of other databases including Web of Science or Cochrane central might have resulted in missing probable relevant records. However, other more common databases which had been checked may cover this issue. ${ }^{40}$ Inclusion of one study reporting mean BNP in patients with prior cardiovascular diseases, due to the availability of fewer studies in this regard, might have made our findings less conclusive for this biomarker. The risk ratio of included studies as well as our final analysis might be indicative of sparse data bias which might be due to some reasons including low prevalence of pre-defined variables or low occurrence of final event per variable. ${ }^{41}$ Moreover, we were not able to categorize patients based on our pre-defined variables to find the exact association. In other words, except for one study, the others did not classify patients in terms of presence/absence of prior cardiovascular diseases for better investigation of the probable effect of these disorders on the incidence or severity of the cardiac injury. Given the lack of reports on the specific method for the diagnosis of acute cardiac injury, we could not analyze the best diagnostic tool in this regard.

In conclusion, our findings suggest that acute cardiac injury is quite common among patients with COVID-19; however, further investigation is required in this regard. Moreover, measurement of specific cardiac biomarkers as prognostic factors, especially among severe patients, must be verified by future studies. Likewise, longterm assessment of this virus could help us with better recognition of probable chronic cardiac complications.

\section{Authors' Contribution}

Study concept and design: MV, DS, MH, NS. Acquisition of data: $\mathrm{MH}, \mathrm{MV}$. Analysis and interpretation of data: MV, MT. Drafting of the manuscript: DS, MV, NR. Critical revision of the manuscript for valuable intellectual content: MV, DS, MH, NS, NR. Statistical analysis: MT. Administrative, technical, and material support: $\mathrm{MH}$, DS, NS. Supervision: DS, MH, NS, NR.

\section{Conflict of Interest Disclosures}

None of the authors had any personal or financial conflicts of interest.

\section{Availability of data and materials}

The datasets generated during and/or analyzed during the current study are not publicly available due to confidential issues but are available from the corresponding author on reasonable request.

\section{Ethical Statement}

Not applicable.

\section{Funding}

No funding has been attributed to this study.

\section{Supplementary Materials}

Supplementary file 1 contains Table S1.

\section{References}

1. Richman DD, Whitley RJ, Hayden FG. Clinical virology. New York: John Wiley \& Sons; 2016.

2. Organization WH. Summary of probable SARS cases with onset of illness from 1 November 2002 to 31 July 2003. Available from: http://www who int/csr/sars/country/table2004_04_21/ en/index html. 2003.

3. Chan KS, Zheng JP, Mok YW, Li YM, Liu YN, Chu CM, et al. SARS: prognosis, outcome and sequelae. Respirology. 2003;8 Suppl(suppl 1):S36-40. doi: 10.1046/j.14401843.2003.00522.x.

4. Badawi A, Ryoo SG. Prevalence of Diabetes in the 2009 Influenza A (H1N1) and the middle east respiratory syndrome coronavirus: a systematic review and meta-analysis. J Public Health Res. 2016;5(3):733. doi: 10.4081/jphr.2016.733.

5. Huang C, Wang Y, Li X, Ren L, Zhao J, Hu Y, et al. Clinical features of patients infected with 2019 novel coronavirus in Wuhan, China. Lancet. 2020;395(10223):497-506. doi: 10.1016/S0140-6736(20)30183-5.

6. Organization WH. Novel Coronavirus (2019-nCoV) Situation report-5, 25 January 2020. Geneva, Switzerland: WHO; 2020.

7. Zhou P, Yang XL, Wang XG, Hu B, Zhang L, Zhang W, et al. A pneumonia outbreak associated with a new coronavirus of 
probable bat origin. Nature. 2020;579(7798):270-273. doi: 10.1038/s41586-020-2012-7.

8. Zhu N, Zhang D, Wang W, Li X, Yang B, Song J, et al. China Novel Coronavirus Investigating and Research Team. A novel coronavirus from patients with pneumonia in China, 2019. N Engl J Med. 2020;382(8):727-733. doi: 10.1056/ NEJMoa2001017.

9. Zheng YY, Ma YT, Zhang JY, Xie X. COVID-19 and the cardiovascular system. Nat Rev Cardiol. 2020;17(5):259-260. doi: 10.1038/s41569-020-0360-5.

10. Zhou F, Yu T, Du R, Fan G, Liu Y, Liu Z, et al. Clinical course and risk factors for mortality of adult inpatients with COVID-19 in Wuhan, China: a retrospective cohort study. Lancet. 2020;395(10229):1054-1062. doi: 10.1016/S01406736(20)30566-3.

11. Hui DS, I Azhar E, Madani TA, Ntoumi F, Kock R, Dar O, et al. The continuing 2019-nCoV epidemic threat of novel coronaviruses to global health-The latest 2019 novel coronavirus outbreak in Wuhan, China. Int J Infect Dis. 2020;91:264-266. doi: 10.1016/j.ijid.2020.01.009.

12. Wang D, Hu B, Hu C, Zhu F, Liu X, Zhang J, et al. Clinical characteristics of 138 hospitalized patients with 2019 novel coronavirus-infected pneumonia in Wuhan, China. JAMA. 2020;323(11):1061-1069. doi: 10.1001/jama.2020.1585.

13. Ruan Q, Yang K, Wang W, Jiang L, Song J. Clinical predictors of mortality due to COVID-19 based on an analysis of data of 150 patients from Wuhan, China. Intensive Care Med. 2020;46(5):846-848. doi: 10.1007/s00134-020-05991-x.

14. Stewart LA, Clarke M, Rovers M, Riley RD, Simmonds $M$, Stewart G, et al. Preferred reporting items for a systematic review and meta-analysis of individual participant data: the PRISMA-IPD statement. JAMA. 2015;313(16):1657-65. doi: 10.1001/jama.2015.3656.

15. Lim W, Qushmaq I, Devereaux P, Heels-Ansdell D, Lauzier F, Ismaila AS, et al. Elevated cardiac troponin measurements in critically ill patients. Arch Intern Med. 2006;166(22):2446-54. doi: 10.1001/archinte.166.22.2446.

16. Downes MJ, Brennan ML, Williams HC, Dean RS. Development of a critical appraisal tool to assess the quality of crosssectional studies (AXIS). BMJ Open. 2016;6(12):e011458. doi: 10.1136/bmjopen-2016-011458.

17. Von Elm E, Altman DG, Egger M, Pocock SJ, Gøtzsche PC, Vandenbroucke JP. The Strengthening the Reporting of Observational Studies in Epidemiology (STROBE) statement: guidelines for reporting observational studies. Int J Surg. 2014;12(12):1495-9. doi: 10.1016/j.ijsu.2014.07.013.

18. Shea BJ, Grimshaw JM, Wells GA, Boers M, Andersson N, Hamel C, et al. Development of AMSTAR: a measurement tool to assess the methodological quality of systematic reviews. BMC Med Res Methodol. 2007;7:10. doi: 10.1186/14712288-7-10

19. Wan X, Wang W, Liu J, Tong T. Estimating the sample mean and standard deviation from the sample size, median, range and/ or interquartile range. BMC Med Res Methodol. 2014;14:135. doi: 10.1186/1471-2288-14-135.

20. Liu M, He P, Liu H, Wang X, Li F, Chen S, et al. Clinical characteristics of 30 medical workers infected with new coronavirus pneumonia [in Chinese]. Zhonghua Jie $\mathrm{He} \mathrm{He}$ Hu Xi Za Zhi. 2020;43(3):209-214. doi: 10.3760/cma.j.is sn.1001-0939.2020.03.014

21. Yang X, Yu Y, Xu J, Shu H, Liu H, Wu Y, et al. Clinical course and outcomes of critically ill patients with SARS-CoV-2 pneumonia in Wuhan, China: a single-centered, retrospective, observational study. Lancet Respir Med. 2020;8(5):475-481. doi: 10.1016/S2213-2600(20)30079-5.

22. Oudit G, Kassiri Z, Jiang C, Liu P, Poutanen S, Penninger J, et al. SARS-coronavirus modulation of myocardial ACE2 expression and inflammation in patients with SARS. Eur J Clin Invest.
2009;39(7):618-25. doi: 10.1111/j.1365-2362.2009.02153.x.

23. Li B, Yang J, Zhao F, Zhi L, Wang X, Liu L, et al. Prevalence and impact of cardiovascular metabolic diseases on COVID-19 in China. Clin Res Cardiol. 2020;109(5):531-538. doi: 10.1007/ s00392-020-01626-9.

24. Lu R, Zhao X, Li J, Niu P, Yang B, Wu H, et al. Genomic characterisation and epidemiology of 2019 novel coronavirus: implications for virus origins and receptor binding. Lancet. 2020 Feb 22;395(10224):565-574. doi: 10.1016/S01406736(20)30251-8.

25. Musher DM, Abers MS, Corrales-Medina VF. Acute infection and myocardial infarction. N Engl J Med. 2019;380(2):171176. doi: 10.1056/NEJMra1808137.

26. Higgins JP, Higgins JA. Elevation of cardiac troponin I indicates more than myocardial ischemia. Clin Invest Med. $2003 ; 26(3): 133-47$.

27. Long B, Long DA, Tannenbaum L, Koyfman A. An emergency medicine approach to troponin elevation due to causes other than occlusion myocardial infarction. Am J Emerg Med. 2020;38(5):998-1006. doi: 10.1016/j.ajem.2019.12.007.

28. Lim W, Cook DJ, Griffith LE, Crowther MA, Devereaux P. Elevated cardiac troponin levels in critically ill patients: prevalence, incidence, and outcomes. Am J Crit Care. 2006;15(3):280-8

29. Metkus TS, Guallar E, Sokoll L, Morrow D, Tomaselli G, Brower $\mathrm{R}$, et al. Prevalence and prognostic association of circulating troponin in the acute respiratory distress syndrome. Crit Care Med. 2017;45(10):1709-1717. doi: 10.1097/ CCM.0000000000002641.

30. Bajwa EK, Boyce PD, Januzzi JL, Gong MN, Thompson BT, Christiani DC. Biomarker evidence of myocardial cell injury is associated with mortality in acute respiratory distress syndrome. Crit Care Med. 2007;35(11):2484-90. doi: 10.1097/01.ccm.0000281852.36573.22.

31. Ammann P, Fehr T, Minder E, Günter C, Bertel O. Elevation of troponin I in sepsis and septic shock. Intensive Care Med. 2001;27(6):965-9. doi: 10.1007/s001340100920.

32. Babuin L, Vasile VC, Perez JAR, Alegria JR, Chai HS, Afessa $B$, et al. Elevated cardiac troponin is an independent risk factor for short-and long-term mortality in medical intensive care unit patients. Crit Care Med. 2008;36(3):759-65. doi: 10.1097/CCM.0B013E318164E2E4.

33. Jeremias A, Gibson CM. Narrative review: alternative causes for elevated cardiac troponin levels when acute coronary syndromes are excluded. Ann Intern Med. 2005;142(9):78691. doi: 10.7326/0003-4819-142-9-200505030-00015.

34. Tanindi A, Cemri M. Troponin elevation in conditions other than acute coronary syndromes [in English]. Ann Biol Clin (Paris). 2017;75(4):411-419. doi: 10.1684/abc.2017.1262.

35. Agewall S, Giannitsis E, Jernberg T, Katus H. Troponin elevation in coronary vs. non-coronary disease. Eur Heart J. 2011;32(4):404-11. doi: 10.1093/eurheartj/ehq456.

36. Anwaruddin S, Lloyd-Jones DM, Baggish A, Chen A, Krauser $D$, Tung $R$, et al. Renal function, congestive heart failure, and amino-terminal pro-brain natriuretic peptide measurement: results from the ProBNP Investigation of Dyspnea in the Emergency Department (PRIDE) Study. J Am Coll Cardiol. 2006;47(1):91-7. doi: 10.1016/j.jacc.2005.08.051.

37. Redfield MM, Rodeheffer RJ, Jacobsen SJ, Mahoney DW, Bailey KR, Burnett JC. Plasma brain natriuretic peptide concentration: impact of age and gender. J Am Coll Cardiol. 2002;40(5):976-82. doi: 10.1016/s0735-1097(02)02059-4.

38. Wang TJ, Larson MG, Levy D, Leip EP, Benjamin EJ, Wilson PW, et al. Impact of age and sex on plasma natriuretic peptide levels in healthy adults. Am J Cardiol. 2002;90(3):254-8. doi: 10.1016/s0002-9149(02)02464-5.

39. Yancy CW, Jessup M, Bozkurt B, Butler J, Casey DE, Drazner $\mathrm{MH}$, et al. 2013 ACCF/AHA guideline for the management of 
heart failure: a report of the American College of Cardiology Foundation/American Heart Association Task Force on Practice Guidelines. J Am Coll Cardiol. 2013;62(16):e147239. doi: 10.1016/j.jacc.2013.05.019.

40. Falagas ME, Pitsouni El, Malietzis GA, Pappas G. Comparison of PubMed, Scopus, web of science, and Google scholar: strengths and weaknesses. FASEB J. 2008;22(2):338-42. doi: 10.1096/fj.07-9492LSF.

41. Greenland S, Mansournia MA, Altman DG. Sparse data bias: a problem hiding in plain sight. BMJ. 2016;352:i1981. doi: 10.1136/bmj.i1981.

42. Cao J, Hu X, Cheng W, Yu L, Tu WJ, Liu Q. Clinical features and short-term outcomes of 18 patients with corona virus disease 2019 in intensive care unit. Intensive Care Med. 2020;46(5):851-853. doi: 10.1007/s00134-020-05987-7.

43. Chen C, Yan J, Zhou N, Zhao J, Wang D. Analysis of myocardial injury in patients with COVID-19 and association between concomitant cardiovascular diseases and severity of COVID-19 [in Chinese]. Zhonghua Xin Xue Guan Bing Za Zhi. 2020;48(7):567-571. doi: 10.3760/ cma.j.cn112148-20200225-00123.

44. Liu Y, Yang Y, Zhang C, Huang F, Wang F, Yuan J, et al. Clinical and biochemical indexes from 2019-nCoV infected patients linked to viral loads and lung injury. Sci China Life Sci. 2020;63(3):364-374. doi: 10.1007/s11427-020-1643-8.

45. Shi S, Qin M, Shen B, Cai Y, Liu T, Yang F, et al. Association of cardiac injury with mortality in hospitalized patients with COVID-19 in Wuhan, China. JAMA Cardiol. 2020;5(7):802810. doi: 10.1001/jamacardio.2020.0950.

46. Peng Y, Meng K, Guan H, Leng L, Zhu R, Wang B, et al. Clinical characteristics and outcomes of 112 cardiovascular disease patients infected by 2019-nCoV [in Chinese]. Zhonghua Xin Xue Guan Bing Za Zhi. 2020;48(6):450-455. doi: 10.3760/ cma.j.cn112148-20200220-00105. 